\title{
Brooke Variance Classification System for DF Bearings ${ }^{1,2}$
}

\author{
E. M. L. Beale
}

(August 22, 1960; revised December 27, 1960)

\begin{abstract}
This paper describes the advantages of having an objective classification system for DF bearings. The Brooke system is described in some detail, and the problems involved in setting up a system on these lines are considered.
\end{abstract}

\section{Introduction}

For many years we used a simple ABCD classification system for HF DF bearings taken with U-Adcock direction finders. This was not entirely satisfactory for various reasons. The fundamental difficulty was that, although in theory the classification depended in a well-defined way on the consistency of the observed bearings, in practice it was largely subjective. The Brooke Variance Classification System is based on the Ross-Barfield system developed during the Second World War and described by Ross [1947]. The present paper is an attempt to describe the system from the point of view of a group that might be considering the introduction of such a system.

Section 2 of this paper reviews the reasons for having a bearing classification system. The sources of error in DF bearings are described briefly in section 3. The history of the Brooke system is reviewed in section 4. Section 5 is devoted to a discussion of the problems involved in introducing a similar system into another network. The solutions found for the Brooke svstem are indicated. Section 6 describes the statistical analysis required to estimate variance components from check bearing data.

\section{Why Does One Need a Classification System?}

The first question is, why does one need a bearing classification system? It is impossible to give a short answer to this question, since it depends on the answer to an even more fundamental question: What use is one trying to make of one's DF bearings? One will presumably plot the bearings reported on any task on a gnomonic chart. One can then either

(1) gaze in wonder at the set of plotted lines, and finally emerge with a point estimate for the position of the transmitter, and a circle of arbitrary radius centered on this point such that the true position "probably" lies within the circle,

or (2) use one of the many unsound plotting methods that have been proposed from time to time,

1 Contribution from Admiralty Research Laboratory, Teddington, Middlesex, England.

${ }_{2}$ Paper presented at the Conference on Transmission Problems Related to High-Frequency Direction Finding, at UCLA, June 21-24, 1960. or (3) use an electronic computer to find a more precise least squares solution, i.e., to find

(a) a Best Point Estimate (or Most Probable Point) defined as the point minimizing the weighted sum of squares of the angular errors, the weights being inversely proportional to the assumed variances of bearing errors at the station concerned, and

(b) a region, normally a circle or a rectangle, approximating to the region where this weighted sum of squares exceeds its minimum value by less than some given constant, such as 4 . The region may be called a 90 percent (say) Probability Region, or Confidence Region. Its purpose is to give an appreciation of the probable accuracy of the point estimate, ${ }^{3}$

or (4) use a method based on some valid approximation to the least squares solution.

In cases (1) and (2) it may be helpful to have some rough measure of the accuracy of any particular bearing, but the traditional ABCD classification based on the consistency of repeated observations, or some other subjective method, may be good enough.

In cases (3) and (4) it is important to have a realistic measure of the probable accuracy of any particular baaring. Ross [1947] wrote

"It has long been customary for bearings to be classified somewhat arbitrarily by the direction-finding operator. This classification, intended to give an indication of the probable reliability of the bearings, is usually based on the quality (sharpness) and steadiness of the minimum. This practice is a legacy from the days when radio communication was conducted chiefly on the long and medium wavelengths, and it was not unreasonable to expect the operator to estimate the reliability of bearings in this way. In the short-wave band, however (20 to $100 \mathrm{~m}$ approximately), where propagation is chiefly controlled by ionospheric reflections, conditions are far from simple. Bearings are generally in a state of continuous variation and it becomes increasingly difficult for an operator to classify them by any more or less intuitive process."

3 The conventional justification for this as a Confidence Region is based on the assumption that all possible bearing lines from a given station are approximately parallel. Beale [1960] provides a method of justifying it even when this approximation is unacceptable. Beale's paper shows that the approximate confidence region is justified if a quantity par so "intrinsic nonlinearity confidence region is justified if a quantity $\mathrm{N}_{\phi}$, called the "intrinsic nonlinearity of the model" is smaller than about 0.1. It seems likely that the DF problem nearly always satisfies this condition; even though one can easily imagine situations with a "narrow base line" when the problem is decidedly nonlinear when expressed in terms of natural parameters, such as the latitude and longitude 0
the transmitter. 
Ross proceeds to describe an objective classification system which is the basis of the Brooke system. The details of such systems will be described in section 4. Meanwhile we should consider another advantage in having a rational and objective classification system: it is almost indispensable to a thorough quantitative analysis of the magnitudes of the various errors arising in practical DF. It is virtually impossible to get a set of observations that is statistically balanced with respect to all relevant factors. So, for example, when studying the effect of distance one must allow for the difference in frequencies of transmitters at different distances; and such a process requires the estimation of variance components due to the different errors making up the observed bearing error.

\section{Sources of Error in HF DF Bearings}

The sources of error in HF DF bearings are discussed by Ross [1947] and Bowen [1955]. They can be summarized as follows:

(1) Instrumental errors due to small errors of antenna balance, and similar causes. These errors can be greatly reduced by a local calibration, but there may be an appreciable residual error varying with radio frequency and direction of incidence (in azimuth and elevation) and from site to site.

(2) Distant-site errors, due to irregularities of, and obstacles on, the terrain around a high-frequency direction finder but outside the near site or calibration area. The values of these errors vary with radio frequency and direction of incidence (in azimuth and elevation). The variances vary from site to site, and tend to decrease as the radio frequency increases.

From our present point of view, errors of types (1) and (2) are similar. They are virtually independent of time. For a given transmitter working on a given frequency the errors may vary a little in that the angle of elevation of the incoming ray will depend to some extent on ionospheric conditions, but there will be an appreciable systematic component of such an error.

(3) Ionospheric lateral deviation errors, due to large scale irregularities in the ionosphere. These errors vary only slowly with time (with periods of the order of tens of minutes). Their variance does not depend on the DF site, but depends strongly on the distance of the transmitter. Not much is known about the effects of time of day and radiofrequency on lateral deviation. It seems possible that errors are somewhat greater at night than by day and somewhat greater on low frequencies than on high frequencies. If so, this is probably due to the increased number of multiple reflections possible at night and on lower frequencies. Large systematic and random effects can be observed around sunrise. Later studies have not essentially changed the views expressed by Ross [1949] on the subject of lateral deviation.

(4) Wave interference errors and polarization errors. These errors may be quite large, but they generally vary rapidly (with periods of the order of seconds). Their effect can therefore be greatly reduced by taking the average bearing (preferably weighted according to signal strength) over a period of $10 \mathrm{sec}$ or longer.

\section{History of the Brooke System}

The Brooke Variance Classification System arose out of the system developed during the Second World War by Mr. W. Ross and Dr. R. H. Barfield, and described by Ross [1947]. The basic assumption underlying the system is that the error in any observed DF bearing is a sum of three uncorrelated errors:

(1) An instrumental and distant site error, whose mean square value can be determined as a function of the radiofrequency and the DF site.

(2) A lateral deviation error, whose mean square value can be determined as a function of the distance from the transmitter to the DF site. This component must of course be estimated at the plotting center.

(3) A wave interference and polarization error. An estimate of the mean square value of this error can be determined from the mean "swing" of the observed bearing over a period of 1 or 2 sec, or from the scatter of repeated observations made at intervals of about 5 or more sec, or both. The distance from the transmitter to the DF site can also be used to throw some light on the probable magnitude of this error, since wave interference due to multipath arrival is apt to be more serious on the more distant targets.

When after the war attention was again directed to the classification of HF DF bearings, the Ross system was examined but considered to be unsatisfactory in its existing form. The users also considered the subjective method of classifying bearings, then in use, to be unsatisfactory. Mr. Norman Brooke, while a member of the Admiralty Department of Operational Research, carried out an investigation into the problem of classifying HF DF bearings, the outcome of which was a proposal to modify the Ross system, and to extend it to apply to visual, as well as aural null, equipment. He found that it was necessary to give the variance components values that were rather larger than those proposed by Ross.

The modified version was found to be satisfactory. It was decided, with Mr. Ross's concurrence, that the system should be called the Brooke system.

Before the system was introduced a statistical analysis was made of a large number of check bearings on known transmitters, in order to derive realistic estimates of all the variance components. Further, it is known that station performance changes to some extent with time, so the analysis of check bearing data continues on a routine basis in order to keep track of these changes. It is found that the variance component depending on the DF station has to be changed by 1 unit (i.e., $1^{\circ}$ squared) from time to time - perhaps once every 2 years on the average. The other variance components are 
reviewed from time to time, but changes have not been found necessary.

So we may conclude that the Brooke system works satisfactorily. The essential reason for this seems to be that the system combines physical theory and empiricism in about the right proportions for our present state of knowledge about HF DF. We certainly do not maintain that no further improvements in the system are possible.

\section{How Could a Similar Classification Sys- tem $\mathrm{Be}$ Introduced Into Another Organ- ization?}

Suppose that some authority has decided that it would be worth introducing an objective bearing classification system on these lines into his $\mathrm{HF}$ DF network, what steps must be taken to implement this decision?

The first task is to define a set of factors on which the variance is assumed to depend. It would seem natural to include

(a) The DF station,

(b) the radiofrequency,

(c) the distance of the transmitter from the station, and

(d) some measure of the quality of the observed bearing.

Some discussion on this last item is perhaps required. A cursory reading of Ross [1947] might give the impression that an objective classification system is only possible if one takes repeated snap observations to determine the bearing. This is not so. The true situation is as follows:

Admittedly if one takes repeated snap observations (which may be mental averages over a period of 1 or 2 sec), then one is likely to obtain a more accurate bearing than if one simply gazes at the display for about $30 \mathrm{sec}$, because experience has shown that it is almost impossible to average mentally over a period of more than about 5 sec. Furthermore, the scatter of the individual snap bearings provides additional information concerning the reliability of the mean bearing. But if the transmitter is not active for long enough to take repeated observations, or if the bearing is required so urgently that there is not time to take and report the results of several snaps, then one can still use an objective classification system based on other factors. Indeed both the original Ross-Barfield system and the Brooke system include means of assessing a variance when it is impossible to obtain more than one snap.

The next task is to postulate a formula for the variance as a function of these factors with a finite number of unknown parameters.

It seems natural to write

$$
B=V_{f}+V_{d}+V_{q},
$$

where B (for Brooke) denotes the total variance (i.e., the total mean square error if one proceeds as if all errors had zero means).
$V_{f}$ denotes a component depending on radiofrequency and the DF station, representing instrumental and distant site errors,

$V_{d}$ denotes a component depending on the distance from the transmitter, representing lateral deviation error, and $V_{q}$ denotes a component depending on the quality of the observed bearing, and the type of equipment and display used, representing wave interference and polarization error, and also observational error due to imperfections in the DF operator.

We must still specify the forms of $V_{f}, V_{d}$, and $V_{q}$ to reduce them to a finite number of parameters. It may be helpful to consider each in turn with reference to the existing Brooke system.

The Brooke system assumes that

$$
V_{f}=a_{j}+b_{j} f,
$$

where $a_{j}$ and $b_{j}$ are parameters estimated separately for each DF station, and $f$ denotes the frequency band. The definition of these frequency bands is being reconsidered. There is some evidence that the variance is not very sensitive to radiofrequency above about $9 \mathrm{Mc} / \mathrm{s}$. The following bands may therefore prove appropriate.

$f=1$ for frequencies between 2.000 and $3.999 \mathrm{Mc} / \mathrm{s}$, $f=2$ for frequencies between 4.000 and $8.999 \mathrm{Mc} / \mathrm{s}$, $f=3$ for frequencies above $9.000 \mathrm{Mc} / \mathrm{s}$.

For the distance component, the Brooke system assumes that

$$
\begin{array}{ll}
V_{d}=0.6 / d^{2} & \text { for } d<0.8, \\
V_{d}=1 & \text { for } 0.8 \leq d<3.5, \\
V_{d}=3 & \text { for } 3.5 \leq d,
\end{array}
$$

where $d$ denotes distance from the transmitter to the DF station, measured in thousands of kilometers.

For the quality component, the Brooke system assumes that

$$
V_{q}=V_{s}+V_{n},
$$

where

$V_{s}=0$ for swings between 0 and $8^{\circ}$,

$=1$ for swings between 9 and $13^{\circ}$,

$=2$ for swings between 14 and $18^{\circ}$,

$=4$ for swings between 19 and $23^{\circ}$,

$=6$ for swings between 24 and $37^{\circ}$,

$=9$ for swings over $38^{\circ}$ or unmeasured,

and $V_{n}=r^{2} / n d_{n}^{2}$,

where $n$ is the number of snap bearings taken, $r$ is the range of these snaps, and $d_{n}$ is the average range of $n$ observations from a Gaussian population with unit variance (tabulated by Tippett [1925]).

The theory underlying the use of these formulas is that $V_{n}$ might be expected to give a valid estimate of the variance of those components of error (due to polarization errors and wave interference) that vary rapidly. But in practice the operator cannot help 
being biased by the knowledge that all his snaps refer to the same transmission. He will therefore tend to make his observations more consistent than they should be, and an additional variance component must be introduced to allow for this. The bias is likely to depend on the difficulty of the task of taking a bearing, which is measured by the average "swing". For an aural null equipment the swing is defined as the width of the arc about the minimum over which there is a just detectable change of signal. For any equipment with a visual display it is defined as the angle through which the instantaneous value of the bearing moves during the time taken to read its mean value for an individual snap. There is of course no logical reason why the formula for $V_{s}$ should be the same for different types of display, even though all the equipments have the same antenna system. But the statistical analysis of check bearings suggests that there is no important difference in this respect between aural null, spinning goniometer, and twin-channel c.r.d.f. displays. ${ }^{4}$

If only one snap is taken, increased values of $V_{d}$ and $V_{s}$ are used to compensate for the absence of the $V_{n}$ term.

Different organizations may find it convenient to estimate $V_{q}$ differently. This must depend on what information the DF operator finds it convenient to report. If he simply reports a letter classification $\left(\mathrm{A}, \mathrm{B}, \mathrm{C}\right.$, or D), then one could put $V_{q}=0$ for an A class bearing, and estimate 3 constants, being the values of $V_{q}$ for $\mathrm{B}, \mathrm{C}$, and $\mathrm{D}$ class bearings respectively.

One might also use the signal strength as an indicator of bearing quality. This does not in fact enter into the existing Brooke system.

Having defined the form of the bearing classification system, one must estimate the parameters. One can get preliminary estimates of these from physical theory and from the values used in existing systems, but these estimates must be corrected (or confirmed) using data obtained by the DF organization in question. These data may be either genuine operational data or check bearing data where the true positions of the transmitters are known to the analyst.

As far as I know, no serious attempt has yet been made to estimate variances from operational data when the true positions of the transmitters are unknown. The use of such data poses the funda-

\footnotetext{
4 There is a small point of some statistical interest about the estimation of $V_{\mathrm{q}}$ 4 There is a small point of some statistical interest about the estimation of $V$ q.
Elementary theory suggests that $\sqrt{ } V_{n}$ is an unbiased estimator of the standard Elementary theory suggests that $\sqrt{ } V_{n}$ is an unbiased estimator of the standard
deviation of errors varving randomly from snap to snap, for a given value of this deviation of errors varving randomly from snap to snap, for a given value of this
standard deviation. But this does not prove that $\sqrt{ } V_{n}$ provides the best estimate of this standard deviation, given the range $r$. Indeed one might expect that a better estimate would be obtained by attaching some weight to the estimate obtained from the long-term average value of this standard deviation-thereby making the used estimate less dependent on $r$. After holding forth on these lines to my colleagues on more than one occasion, I eventually persuaded them to do a detailed analysis of the check bearing data to investigate this point. They found that, if anvthing, the variance component $V_{n}$ should be made more de. pendent on $r$. It has been suggested that this is because the dividing factor pendent on $r$. It has been suggested that this is because the dividing factor $d_{n^{2}}$ is too large because the error distribution is not normal. (And indeed even from a normal population a smaller dividing factor is required to give an unbiased estimate of the variance as opposed to the standard deviation.) But I prefer to regard this as suggesting that the range has additional value as an indicator of the quality of the bearing. More light could be thrown on this point by an analysis
of the individual snap observations, but it is of little operational importance.
}

mental difficulty that one has to lean rather heavily on the assumption that the bearing errors are uncorrelated. If one is prepared to accept this assumption, then it is fairly easy to estimate the absolute values of the variances of a set of DF stations if we know their relative variances. For in this case we can determine the true Best Point Estimate; i.e., the hypothetical position of the transmitter minimizing the sum of squares of the bearing errors divided by their variances. We can then use the fact that (if the error distributions are normal with zero mean) the minimum value of this sum is distributed as $\chi^{2}$ with $n$-2 degrees of freedom, where $n$ is the number of DF stations contributing to a task. In particular, the mean value of this sum is $n-2.5$

In practice the relative variances are unlikely to be known. The problem then becomes considerably more complicated. It is discussed in a companion paper, Beale [1961].

It is much easier to estimate variances from check bearings, provided satisfactory check bearings can be obtained.

Check bearings may be used both to verify that the equipment is functioning properly and to indicate the probable accuracy of the DF station on operational tasks. A check bearing program to check the equipment does not need to be controlled nearly as carefully as a check bearing program to estimate accuracy. Bowen [1955] writes

"The use of check bearings on known transmitters in establishing the performance of a high-frequency directionfinder is a widely accepted technique. It has been found, however, that two problems present themselves:

(a) There is difficulty in arranging a programme in which the radio frequencies, ranges, azimuths, propagation paths, transmitter powers and other parameters are adequately sampled and distributed similarly to those which will define the normal task of the direction finder.

(b) Unless great care is taken to hide the identity of the transmission from the operator, both as regards its true bearing and its identity with previous transmissions, considerable operator bias will occur.

"The methods by which these problems are overcome will generally be peculiar to the task with which the d.f. organization is concerned. It may be that (a) will require the deliberate setting-up of a transmitting system, although careful selection of known trans nitters may obviate this; (b) can be overcome either by careful organization of the way in which check-bearing tasks are fed to the operator, or by a system of random scale displacements at the direction-finder."

Even if one fails to overcome these problems completely, it would seem unreasonable to use this as an excuse for not introducing an objective classification system. Indeed the results from an objective system should be very much better than those derived from overall station performances based on such data - since the system will give some rational method of extrapolating to conditions met only rarely in the check bearing program.

T'he statistical problems involved in estimating variance components from check bearings are discussed in the next section.

${ }^{5}$ Provided, as will usually be the case, that the quantity $N_{\phi}$ introduced by Beale [1960] is small. 


\section{Estimating Variance Components From Check Bearing Data}

The fundamental statistical problem in this work can be expressed as follows:

Given $n$ independent observations, each from a normal population with zero mean, such that the $i$ th observation $x_{i}$ has a variance

$$
B_{i}=\sum_{j=1}^{p} \alpha_{i j} v_{j}
$$

where the $\alpha_{i j}$ are all known but some at least of the $v_{j}$ are unknown, estimate the unknown $v_{j}$.

Before showing how to solve this problem, I should perhaps indicate its relevance. The quantity $x_{i}$ represents the actual error in the $i$ th bearing observation, $B_{i}$ represents its assumed variance, and the $v_{j}$ represent the parameters in the classification system. For example, suppose that we have a simple model with one component that can be assumed known, and unknown components depending on the radiofrequency and quality, assumed to depend on 4 parameters, such that

$$
V_{f}=v_{1}+f v_{2},
$$

where $f$ denotes frequency band, either 1,2 or 3 , and

$$
\begin{aligned}
& V_{q}=v_{3} \text { if the swing }<8^{\circ}, \\
& V_{q}=v_{4} \text { if the swing } \geq 8^{\circ} .
\end{aligned}
$$

Now suppose that the first few check bearings have the following characteristics:

$\begin{array}{cccc}\text { Bearing No. } \begin{array}{c}\text { Known Vari- } \\ \text { ance Component }\end{array} & \begin{array}{c}\text { Frequency } \\ \text { Band }\end{array} & \text { Swing } \\ 1 & 6 & 1 & 3^{\circ} \\ 2 & 3 & 2 & 13^{\circ} \\ 3 & 5 & 3 & 7^{\circ}\end{array}$

etc

Then, if we write formally $v_{5}=1,(6.1)$ is satisfied with

$$
\begin{aligned}
& \alpha_{11}=1, \alpha_{12}=1, \alpha_{13}=1, \alpha_{14}=0, \alpha_{15}=6, \\
& \alpha_{21}=1, \alpha_{22}=2, \alpha_{23}=0, \alpha_{24}=1, \alpha_{25}=3, \\
& \alpha_{31}=1, \alpha_{32}=3, \alpha_{33}=1, \alpha_{34}=0, \alpha_{35}=5,
\end{aligned}
$$

the parameters to be estimated being $v_{1}, v_{2}, v_{3}$, and $v_{4}$.

We assume that our bearing errors are measured ni degrees. Variances are therefore measured in degrees squared.

Now $B_{1}$ is intended to represent the mean value of $x_{i}^{2}$, so it seems natural to try to minimize the sum of squares of the deviations of the $x_{i}^{2}$ from the corresponding $B_{i}$. But we must remember that the expected magnitudes of these deviations depend on the variances of the $x_{i}^{2}$. In fact if $x_{i}$ is normally distributed the variance of $x_{i}^{2}$ is $2 B_{i}^{2}$. It therefore seems reasonable to try to minimize

$$
\sum_{i=1}^{n} w_{i}\left(x_{i}^{2}-B_{i}\right)^{2}
$$

where the weights $w_{i}$ are regarded as constants but where their numerical values are given by

$$
w_{i}=1 / \mathrm{B}_{i}^{2}
$$

Differentiating (6.2) with respect to $v_{j}$, regarding $w_{i}$ as constant, we have, using (6.1)

$$
\sum_{i=1}^{n} w_{i} \alpha_{i j}\left(x_{i}^{2}-B_{i}\right)=0
$$

for each $j$ such that $v_{j}$ is to be estimated.

In particular if for some $j, \alpha_{i j}$ is always either 0 or 1 , the corresponding equation from (6.4) becomes

$$
\Sigma_{i}^{\prime} w_{i}\left(x_{i}^{2}-B_{\imath}\right)=0,
$$

where $\Sigma_{i}^{\prime}$ denotes summation over all observations such that $\alpha_{i j}=1$.

The problem is therefore to find estimates $\hat{v}_{1}$ for the unknown $v_{j}$ satisfying (6.1), (6.3) and (6.4). This will have to be done iteratively. Choose trial estimates for the $v_{j}$, compute the corresponding values of $B_{i}$ from $(6.1)$, and hence compute the weights $w_{i}$ from (6.3), substitute these values of $w_{i}$ in (6.4) and hence estimate new values of the $v_{j}{ }^{6}$ The whole cycle can be repeated if necessary, but it should be remembered that small (relative) errors in the $w_{i}$ will be of little significance.

It can easily be shown that these estimates are in fact maximum likelihood estimates for the $v_{j}$. The above derivation is less rigorous but seems more intuitive. $^{7}$

One important proviso should be made. It is undesirable to have weights for individual bearings of the same order of magnitude as the sum of the weights for all other bearings put together. In the analyses for the Brooke system, this is avoided by putting $w_{i}=1 / 2$ whenever $B_{i}<\sqrt{2}$. So the used version of $(6.3)$ reads

$$
w_{i}=\min \left(1 / 2,1 / B_{i}^{2}\right) .
$$

It is of interest to consider the probable accuracy of the final variance estimates. It is easy to find the variance of an individual component, say $v_{k}$, if we assume that all the other components are known exactly, and also that each $w_{i}$ equals the reciprocal of the square of the true variance of the

\footnotetext{
${ }^{6}$ In practice it may be more convenient to improve the estimates of the parameters referring to one variance component, say $V_{f}$, assuming that the others have their present trial values. This reduces the number of equations that have to be solved simultaneously.

7 Some statisticians may point out that for this problem the method of maximum likelihood gives an "inadmissible" estimator, since it may produce negative variance components. With a fair-sized sample this is unlikely to happen; but, if it does, one must replace the negative component by some more plausible value and re-estimate the other components accordingly.
} 
corresponding $x_{i}$. For (6.4) then becomes

$$
\sum_{i=1}^{n} w_{i} \alpha_{i k}\left(x_{i}^{2}-\sum_{j=1}^{p} \alpha_{i j} v_{j}\right)=0,
$$

so that the estimate $\hat{v}_{k}$ of $v_{k}$ is given by

$$
\hat{v}_{k}=\sum_{i=1}^{n} w_{i} \alpha_{i k}\left(x_{i}^{2}-\sum_{j \neq k} \alpha_{i j} v_{j}\right) / \Sigma w_{i} \alpha_{i k}^{2} .
$$

Now var $x_{i}^{2}=2 B_{i}^{2}$, and $w_{i}=1 / B_{i}^{2}$, so

$$
\begin{aligned}
\operatorname{var} \hat{v}_{k} & =\sum_{i=1}^{n}\left(\frac{\alpha_{i k}}{B_{i}^{2}}\right)^{2} \cdot 2 B_{i}^{2} /\left(\sum_{i=1}^{n} \frac{\alpha_{i k}^{2}}{B_{i}^{2}}\right)^{2} \\
& =2 / \sum\left(\frac{\alpha_{i k}^{2}}{B_{i}^{2}}\right) \\
& =2 / \sum w_{i} \alpha_{i k}^{2} .
\end{aligned}
$$

If $\alpha_{i k}=0$ or 1 for all $i,(6.5)$ reduces to

$$
\operatorname{var} \hat{\imath}_{k}=2 / \Sigma_{k}^{\prime} w_{i}
$$

where $\Sigma_{k}^{\prime}$ denotes summation over those check bearings for which $\alpha_{i k}=1$, i.e., for which the given variance component is relevant.

Errors in estimating the weights $w_{i}$, and also errors in the other variance components, will not normally have a major effect on the variance of $\hat{v}_{k}$. On the other hand the fact that the observations may not be strictly independent, and that the error distribution may not be strictly normal, may have a considerable effect on the validity of these formulas. Our experience suggests that, presumably because of these causes, formulas (6.5) and (6.6) underestimate the variances of the $\hat{v}_{k}$ by a factor of about 2 . The formulas

$$
\operatorname{var} \hat{v}_{k} \approx 4 / \Sigma w_{i} \alpha_{i k}^{2} \text {, or } \operatorname{var} \hat{v}_{k} \approx 4 / \Sigma_{k}^{\prime} w_{i},
$$

are therefore recommended in practice.

There is one pecularity about these variance component estimates that one should be aware of, although it does not affect the operation of the system in any way. There is a degree of indeterminacy about the system in that a constant can be subtracted from all values of, say, $V_{d}$ and added to all values of, say, $V_{f}$ without altering any of the Brooke variances. The only restriction is that none of the variance components can ever be negative. Our practice is to put the minimum values of $V_{d}$ and $V_{q}$ equal to zero, and to put the "unattached variance", into $V_{f}$. This is purely for operational convenience. In fact we believe that the minimum value of the effect of lateral deviation, which is represented by $V_{d}$, is about $1^{\circ}$ squared, as indicated in section 5 above.

We have now considered the estimation of variance components in the first instance.
The other vital element in an objective bearing classification system is a method of keeping the system up-to-date. For routine corrections to be timely and not unduly subject to sampling fluctuations, the corrections should be based on as simple a statistical model as possible. We therefore assume that all variances of bearings taken at a particular station are underestimated by a constant small amount. The best estimate of this amount is then approximately

$$
\Sigma w_{i}\left(x_{i}^{2}-B_{i}\right) / \Sigma w_{i}
$$

where summation extends over all bearings taken at this station. This quantity is called the "apparent correction", short for "apparent Brooke variance correction", and its variance is approximately $4 / \Sigma w_{i}$. If we wait until $\Sigma w_{i} \geq 20$, the variance of the apparent correction is less than about 0.2 , so the correction is unlikely to be in error by as much as one unit. Nevertheless the apparent correction is rounded down in absolute value to the nearest unit, since some weight should be given to the fact thatif the system has been running for some timeprevious data suggested that no correction was necessary.

In practice this works as follows. For each check bearing the "weight" $w_{i}$ and the "indicator" $w_{i}\left(x_{i}^{2}-B_{i}\right)$ are recorded, and these are summed for all check bearings taken in a month at each station. These data are inspected and combined with those for enough previous months to make $\Sigma w_{i} \geq 20$ for the station in question. Then $\Sigma w_{i}\left(x_{i}^{2}-B_{i}\right) / \Sigma w_{i}$, rounded down in absolute value to the nearest unit, is added to all values of $V_{f}$ for this station; i.e., to the value of $a_{j}$ in the formula $V_{f}=a_{j}+b_{j} f$.

After any adjustment to the value of $a_{j}$, the monthly totals of $\Sigma w_{i}\left(x_{i}^{2}-B_{i}\right)$ must obviously be reduced by $\Sigma w_{i}$ multiplied by the addition to $a_{j}$ before they are combined with subsequent monthly totals to decide whether further changes should be made. But it should never be necessary to recompute the $B_{i}$ and $w_{i}$ for individual bearings.

In practice our estimated variance components are not subject to frequent oscillations.

In addition to these routine adjustments to the overall variances for each station, it is important that the other variance components should be reviewed from time to time, by computing apparent corrections from the formula $\Sigma w_{i}\left(x_{i}^{2}-B_{i}\right) / \Sigma w_{i}$ with summation extending over all check bearings associated with a particular value of the component under review. The desirability of introducing further terms into the expression for the variance can be tested in the same way.

This may be as good a place as any to mention the problems of systematic errors and wild bearings. Many DF stations exhibit small systematic errors in their bearings. These have been studied for some time, but corrections to allow for them are not applied because the physical basis of the errors is not understood. If the systematic error is ignored, one is acting as if the error distribution had mean zero. 
and the Brooke variance is really an estimate of the mean square error.

DF stations are apt to produce a certain number of wild bearings that are a long way off the true bearing, and which one hopes to be able to reject in plotting by their inconsistency with the other bearings. Obviously one does not want to include these in one's check bearing data for the determination of variances. But the problem is, how large must the error be before one is justified in rejecting the bearing as wild on the grounds that it would probably be recognized as such even when the true target position was unknown? This is not an easy problem. The proper answer presumably depends on the variance of nonwild observations, and on the number, location, and accuracy of the other DF stations in the organization working on the same tasks. The rejection of bearing errors greater than 10 or $15^{\circ}$ may often be reasonable.

Although it has fallen to me to present this account of the Brooke system, I hope I have made it clear that most of the credit belongs elsewhere. I have been able to refer to some of the relevant work by Messrs. Ross, Brooke, and Bowen; but many people have contributed in various ways - notably those who organize the check bearing program, and collate and analyze the data.

\section{References}

Beale, E. M. L., Confidence regions in nonlinear estimation, J. Roy. Stat. Soc. (B) 22, 41-88 (1960).

Beale, E. M. I., The estimation of variances of position lines from fixes with unknown target positions, J. Research NBS 65D (Radio Prop.)

(May-June 1961).

Bowen, K. C., Sources of error in U-Adcock high-frequency direction-finding, Proc. I.E.E. 102 Pt. B, 529-532 (1955).

Ross, W., The estimation of the probable accuracy of high frequency radio direction-finding bearings, Journal I.E.E. 94 Pt. 3Ä, 722-726 (1947).

Ross, W., Lateral deviation of radio waves reflected at the ionosphere, DSIR Radio Research Special Rept. No. 19 (1949).

Tippett, L. H. C., On the extreme individuals and the range of samples taken from a normal population, Biometika $\mathbf{1 7}$ 364-387 (1925).

(Paper 65D3-128) 